\title{
Characterization and Modelling of Circular Piezoelectric Micro Speakers for Audio Acoustic Actuation
}

\author{
J. Mendoza-López, S. Sánchez-Solano, and J. L. Huertas-Díaz \\ Instituto de Microelectrónica de Sevilla-Centro Nacional de Microelectrónica, c/Américo Vespucio s/n., 41092 Sevilla, Spain \\ Correspondence should be addressed to J. Mendoza-López, mendoza@imse-cnm.csic.es
}

Received 23 September 2011; Accepted 30 October 2011

Academic Editors: J. Hu, D. Kalempa, and X. Yang

Copyright (๑) 2012 J. Mendoza-López et al. This is an open access article distributed under the Creative Commons Attribution License, which permits unrestricted use, distribution, and reproduction in any medium, provided the original work is properly cited.

\begin{abstract}
A study of circular piezoelectric micro speakers is presented for applications in the audio frequency range, including values for impedance, admittance, noise figures, transducer gain, and acoustic frequency responses. The micro speakers were modelled based on piezoelectric micro ultrasonic transducer (pMUT) design techniques and principles. In order to reach the audio frequency range, transducer radii were increased to the order of one centimetre, whilst piezoelectric layer thicknesses ranged the order of several $\mu \mathrm{m}$. The micro actuators presented might be used for a variety of electroacoustic applications including noise control, hearing aids, earphones, sonar, and medical diagnostic ultrasound. This work main contribution is the characterization of the design space and transducer performance as a function of transducer radius, piezoelectric layer thickness, and frequency range, looking towards an optimized fabrication process.
\end{abstract}

\section{Introduction}

Multimedia systems and components such as micro speakers are elements of great interest for the research community, driven by the needs of mobile phone, entertainment, laptop, tablets, and computer industries. It has been long time since "full range" multimedia micro speakers are trying to be developed, often finding design conflicts limited by fundamental physics. For example, the concept of miniaturization is in conflict with the idea of full-range: the smaller the radiating source, the higher the operating frequency range. Low frequencies and therefore large wavelengths require big source sizes which mean large loudspeaker diaphragms and piston excursion.

There are usually many more conflicting requirements and tradeoffs found in the design of high sound pressure level output, low noise and distortion, full audio range, omnidirectional, and minimum size transducers.

Some studies concerning micro speakers of the moving coil type have been reported in [1-4]. Within this type of speakers, performance was improved after modifying magnetic characteristics [2], optimizing diaphragm pattern design via a finite element approach [5] or introducing a combined permanent magnet [4]. Although moving-coil is a well-matured technology, and probably best for mid- or low-frequency range loudspeaker units, the voice coil and the magnet do produce coupling interactions and nonlinearities difficult to deal with $[6,7]$, introducing complexity in the design.

A different approach to micro speaker development is the piezoelectric type, as reported amongst others in [8-12]. Driven not only by applications in the audio field but most importantly by biomedical and underwater ultrasound applications and requirements, the piezoelectric micro ultrasonic transducer (pMUT) has been widely described in [1317]. The fabrication details are by now well established together with their performance characteristics. Advantages of piezoelectric type transducers include smaller size, lower costs, batch fabrication, and integration with CMOS, which may allow for fabrication with required electronics such as amplifier or other signal conditioning circuits. Their main disadvantage is the difficulty of finding foundry standard processes which include piezoelectric layers for research and inexpensive prototyping, in contrast with the other main 


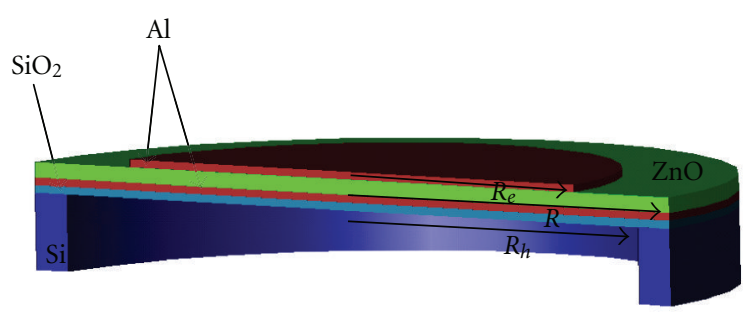

Figure 1: Schematic view of simulated actuator. Layers, from top to bottom: top electrode, piezoelectric layer(s), bottom electrode, oxide layer, and Si-structural layer.

types of micro ultrasonic transducers: the capacitive type (cMUT), which can be fabricated with multiuser MEMS processes [18] or simply with standard CMOS foundry processes [19]. Impedance matching with radiating media properties, in our case air, is also an important issue: the acoustic impedance of air $\left(400 \mathrm{~kg} / \mathrm{m}^{2} \mathrm{~s}\right)$ is considerably smaller than that of piezoelectric materials commonly used in ultrasound imaging $\left(30 \times 10^{6} \mathrm{~kg} / \mathrm{m}^{2} \mathrm{~s}\right)$, for which matching techniques have to be employed.

In this paper, the techniques used for pMUT design and modelling which comprise some of radio-frequency (RF) system analysis are extended to the audio acoustic frequency range for the numerical analysis and characterization of a piezoelectric unimorph micro speaker. A design is presented by which the actuating membrane is made only of isolating $\mathrm{SiO}_{2}$ and a layer of nearly 100 micron of $\mathrm{ZnO}$ film, electroded on both sides. The resulting transducer, operating in the thickness mode, may be used for a variety of sound and vibration applications including active noise control, parametric arrays, beamforming arrays, and vibration sensors. Results are presented showing the most important design tradeoffs and their consequences on performance.

\section{Transducer Design}

The transducer presented is a piezoelectric unimorph with a $\mathrm{Si}-\mathrm{SiO}_{2}-\mathrm{Al}-\mathrm{ZnO}-\mathrm{Al}$ layer stack, as shown in Figure 1, in thickness mode excitation. In order to operate at audio frequencies, transducer dimensions have to be increased by several orders of magnitude from those corresponding to micro transducers intended for ultrasonic radiation. Transducer radii range from $0.75 \mathrm{~mm}$ to $1.25 \mathrm{~cm}$, and layer thicknesses range between $10 \mu \mathrm{m}$ and $0.5 \mathrm{~mm}$.

The described devices are intended to be fabricated in a post-CMOS technology by stacking layers of piezoelectric material on a mechanical silicon substrate. A schematic view of the layer stack and geometric variables is shown in Figure 1. The design parameter ranges are specified in Table 1.

\section{Theoretical Background}

3.1. Piezoelectric Actuation. Following the IEEE Standard on Piezoelectricity [20], the linear piezoelectric constitutive equations relating the electrical field $\mathbf{E}_{i=1-3}$ in the case of actuators or the spatial polarization vector $\mathbf{P}_{i=1-3}$ in the case
TABLE 1: Transducer design variables.

\begin{tabular}{lcccc}
\hline Meaning & Symbol & Units & Min. value & Max. value \\
\hline Sustrate thickness & $t_{\text {sus }}$ & $\mathrm{mm}$ & 1 & 10 \\
Si thickness & $t_{\mathrm{Si}}$ & $\mathrm{mm}$ & 1 & 10 \\
$\mathrm{SiO}_{2}$ thickness & $t_{\mathrm{SiO}_{2}}$ & $\mu \mathrm{m}$ & 1 & 100 \\
Electrode thickness & $t_{\mathrm{el}}$ & $\mu \mathrm{m}$ & 1 & 100 \\
ZnO thickness & $t_{\text {piezo }}$ & $\mu \mathrm{m}$ & 1 & 500 \\
Radius & $R$ & $\mathrm{~mm}$ & 0.75 & 12.5 \\
Top electrode radius & $R_{e}$ & $(\%$ of $R)$ & 1 & 100 \\
Bottom hole radius & $R_{h}$ & $(\%$ of $R)$ & 1 & 100 \\
\hline
\end{tabular}

of sensors to the corresponding longitudinal and shear components of the mechanical stress $\mathbf{T}_{j=1-6}$ or strain $\boldsymbol{S}_{j=1-6}$ tensors are

$$
\begin{gathered}
\mathbf{S}_{j=1-6}=\sum_{i=1}^{3} \mathbf{d}_{i j} \mathbf{E}_{i=1-3}+\sum_{h=1}^{6}\left(\frac{1}{\mathbf{C}_{j h}^{E=c t e}}\right) \mathbf{T}_{j}, \\
\mathbf{T}_{j=1-6}=-\sum_{i=1}^{3} \mathbf{e}_{i j} \mathbf{E}_{i=1-3}+\sum_{h=1}^{6} \mathbf{C}_{j h}^{E=c t e} \boldsymbol{S}_{j},
\end{gathered}
$$

where $\mathbf{d}_{i j}[\mathrm{~m} / \mathrm{V}]$ and $\mathbf{e}_{i j}\left[\mathrm{C} / \mathrm{m}^{2}\right]$ are the piezoelectric $3 \times 6$ constants of the piezoelectric material, and the indexes $i$ and $j$ denote polarization and actuation direction, respectively. $\mathrm{C}_{j h}$ represents the $6 \times 6$ elastic stiffness of the piezoelectric material under constant electric field, and it relates the two piezoelectric constant types following the expression:

$$
\mathbf{e}_{m h=1-6}=\sum_{h=1}^{6} \mathbf{C}_{j h}^{E=c t e} \mathbf{d}_{m j} .
$$

The dielectric polarization $\mathbf{P}_{i=1-3}$ of the piezoelectric material is expressed as

$$
\begin{aligned}
\mathbf{P}_{i=1-3} & =\mathbf{D}_{i=1-3}-\varepsilon_{0} \mathbf{E}_{i=1-3} \\
& =\sum_{j=1}^{3}\left(\varepsilon_{i j}-\varepsilon_{0}\right) \mathbf{E}_{i=1-3}+\sum_{j=1}^{6} \mathbf{e}_{i j} \mathbf{S}_{j=1-6},
\end{aligned}
$$

with $\varepsilon_{i j}$ (units of $\mathrm{F} / \mathrm{m}$ ) and $\mathbf{D}_{i}$ (units of $\mathrm{C} / \mathrm{m}^{2}$ ) representing the $3 \times 3$ permittivity matrix and electric displacement vector, respectively, and $\varepsilon_{0}$ is the permittivity of free space. Equations (1) to (4) are the existing fundamental piezoelectric constitutive equations which describe the membrane behaviour.

3.2. Transducer as a Two-Port Network. For the electromechanical analysis of our interest, a piezoelectric actuator can be described as a linear two-port network (Figure 2), for which the following relations hold between electrical and mechanical inputs and outputs for harmonic excitation under standard electromechanical analogy:

$$
\left[\begin{array}{c}
\mathbf{F} \\
-\mathbf{u}
\end{array}\right] \equiv\left[\begin{array}{c}
\mathbf{V}_{o} \\
\mathbf{I}_{o}
\end{array}\right]=\left[\begin{array}{ll}
Z_{11} & Z_{12} \\
Z_{21} & Z_{22}
\end{array}\right] \cdot\left[\begin{array}{c}
\mathbf{V}_{i} \\
\mathbf{I}_{i}
\end{array}\right]
$$




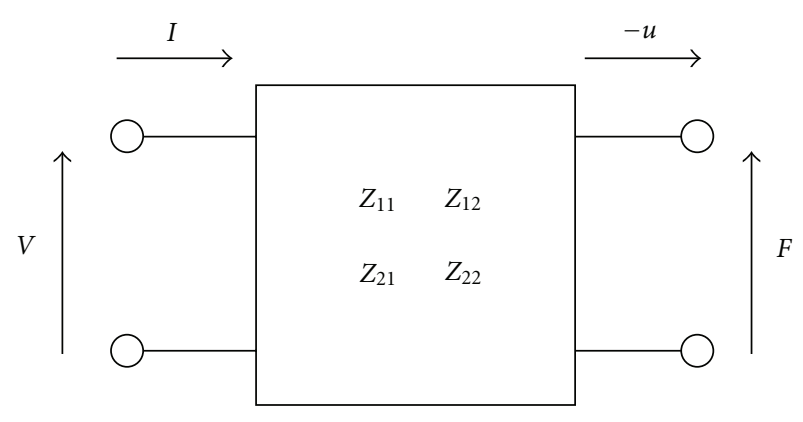

FIgure 2: The electromechanical transducer represented schematically as a two-port network.

where $\mathbf{V}$ and I denote complex voltage and current and the subscripts $i$ and $o$ denote input and output, respectively. $\mathbf{F}$ and $\mathbf{u}$ denote complex force and particle velocity on the front face of the transducer, and the matrix $\mathbf{Z}$ containing elements $Z_{11}, Z_{12}, Z_{21}$, and $Z_{22}$ is the complex impedance matrix for the transducer viewed as a two-port network. By modelling the transducer with a numerical simulation environment such as Simulation Program with Integrated Circuits Emphasis (SPICE), data for the impedance matrix or particle velocity amongst other quantities can be obtained. For a more detailed analytical two-port, lumped-element modelling of a piezoelectric unimorph composite circular plate, see [21]. In this work, the matrix $\mathbf{Z}$ was computed with Spectre after modelling the actuator with the commercial MEMS software MEMS+ by Coventor Inc.

3.3. Vibration Problem. In order to relate the acoustics of the device to the intrinsic material properties of the membrane, the problem has to be tackled from an electromechanoacoustical perspective. In the mechanical domain considering vibrating plates, Euler's flexural equation of motion for a forced circular plate with clamped edges would apply. According to our results and following previous work on vibrating piezoelectric circular diaphragms [22], piezoelectric micromachined devices behave more like membranes (resonance frequencies proportional to $R^{-1}$ ) than like thin plates (resonance frequencies proportional to $R^{-2}$ ). Therefore the problem can be formulated as a forced circular membrane with clamped edges, for which the equation of motion is

$$
T \nabla^{2} w(\mathbf{r}, t)+\rho_{0} \frac{\partial^{2} w(\mathbf{r}, t)}{\partial t^{2}}=f(\mathbf{r}, t),
$$

where $w(\mathbf{r}, t)$ is the membrane's displacement at a point pointed by vector $\mathbf{r}$ and instant $t, f(\mathbf{r}, t)$ is the forcing function at any point pointed by $\mathbf{r}$ and instant $t, T$ is the tension on the membrane, $\nabla^{2}$ is the Laplacian operator, and $\rho_{0}$ is the material density.

Solving (6) for $w(\mathbf{r}, t)$ with the appropriate boundary conditions corresponding to clamped edges gives an expression for the displacement at any point of the membrane at any instant $t$, given a known forcing function. The solution to (6) is usually not easy to compute and analytical solutions might not exist depending on the geometry and boundary conditions. Finite integral transforms might be obtained [23] or alternatively numerical solutions can be computed by the finite element and boundary element methods [24].

In our case, the vibration of the membrane was computed numerically by means of the commercial design and visualization software MEMS+.

3.4. Acoustic Radiation. The acoustic pressure field $p(\mathbf{r}, t)$ at any point $\mathbf{r}$ and time instant $t$ generated in free field by a radiating surface $S$ with centre at the coordinates origin might be obtained from the Rayleigh integral [25] as

$$
p(\mathbf{r}, t)=\frac{j \omega \rho_{0}}{2 \pi} e^{j \omega t} \int_{\mathbf{S}} \frac{\mathbf{u} \cdot e^{-j k r}}{r} \mathbf{d} \mathbf{S},
$$

where $\omega$ is the angular frequency, $\rho_{0}$ is the fluid density, $k$ is the wavenumber, $r=|\mathbf{r}|$ is the scalar distance from the origin to the listening point, $\mathbf{u}$ is the complex particle velocity, and $j$ is the complex unit number. In our case we can use the particle velocity $\mathbf{u}$ calculated numerically from (5), to obtain a good approximation of the radiated sound pressure field.

When integrated over a circumference of radius $R$ accounting for the transducer's diaphragm surface, Rayleigh integral yields

$$
p(\mathbf{r}, t)=\mathbf{I}_{0} \frac{e^{j(\omega t-k r)}}{r} D(\theta)
$$

where $\mathbf{I}_{\mathbf{0}}$ is the complex output current numerically calculated after $(5)$ and $D(\theta)$ represents the directivity function for a flat circular piston of radius $R$ on an infinite baffle at an off-axis angle $\theta$, given by

$$
D(\theta)=\left|\frac{2 J_{1}(k R \sin (\theta))}{k R \sin (\theta)}\right|,
$$

with $J_{1}$ being the Bessel function of the first kind, $k=2 \pi / \lambda=$ $\omega / c$ the wavenumber, $\lambda$ the wavelength, and $\omega$ the angular frequency.

Therefore the acoustic output of the transducer can be modelled by a directivity function corresponding to a flat circular piston multiplying the current frequency response characteristic of the transducer, together with a complex exponential in time and distance and a $1 / r$ decay factor [26].

\section{Results}

The commercial software MEMS+ from Coventor Inc. was used for transducer design and visualization in conjunction with Cadence Virtuoso as a numerical calculation engine. An $\mathrm{AC}$ analysis was performed to calculate the frequency response voltages and currents. An s-parameter analysis was used for impedance, admittance, noise figure, and transducer gain calculation. Both analyses were computed between $100 \mathrm{~Hz}$ and $100 \mathrm{kHz}$.

As it can be seen in Figure 3, impedance magnitude goes down and impedance resonance frequency lower as radius increases. Admittance behaviour is obviously the opposite: magnitude goes up and resonance frequency also goes down in the same fashion as radius increases. To illustrate how resonance frequencies vary as a function of radius or piezoelectric layer thickness, the values of the resonance peaks can 


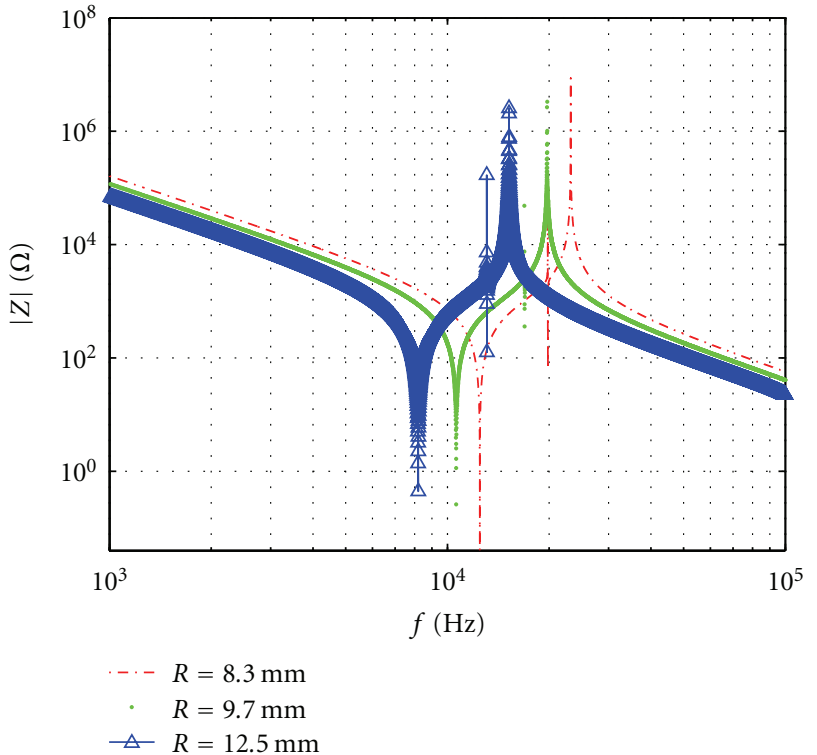

(a)

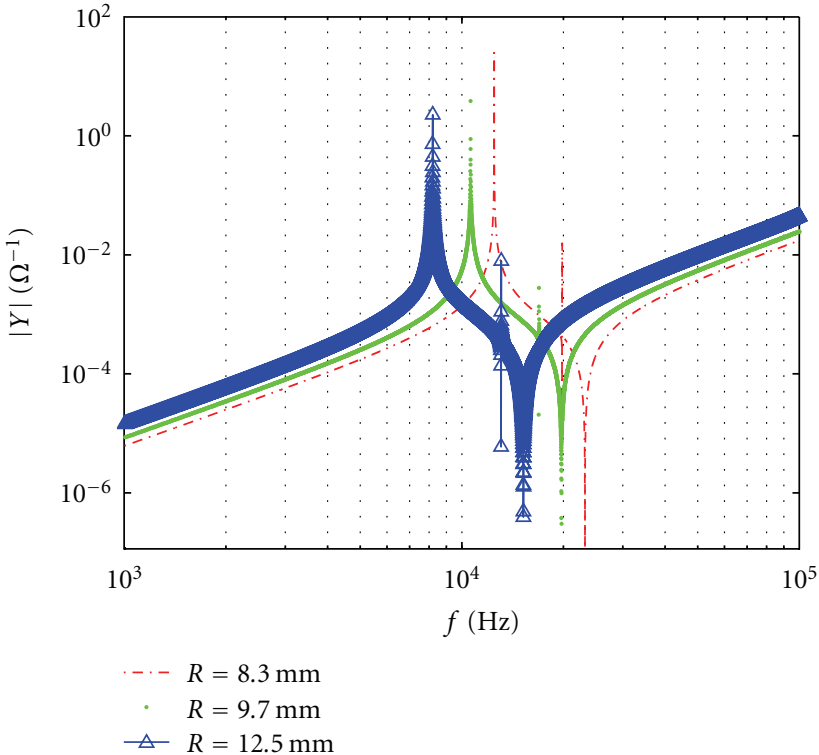

(b)

Figure 3: Transducer impedance magnitudes (a) and admittance magnitudes (b) calculated for three different radii, all of them for a piezoelectric layer thickness of $80 \mu \mathrm{m}$.

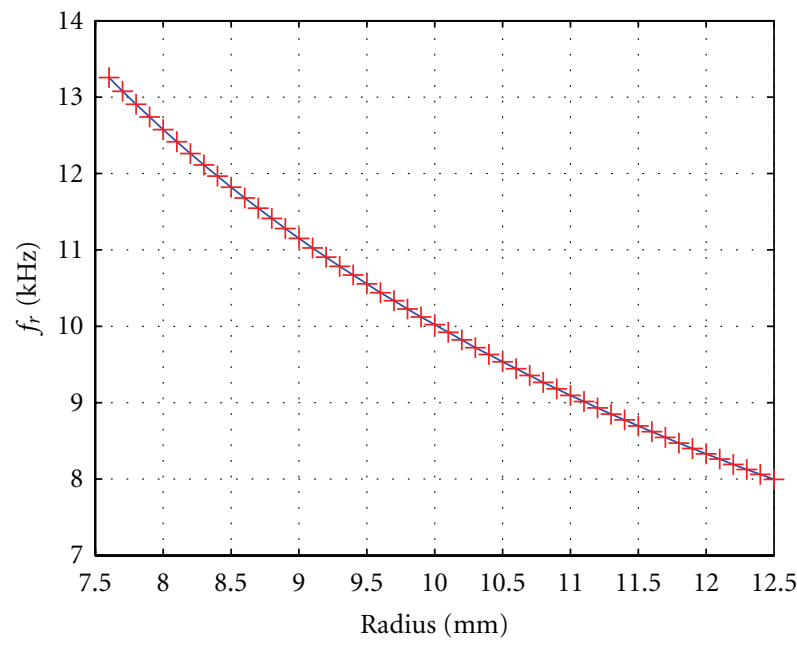

(a)

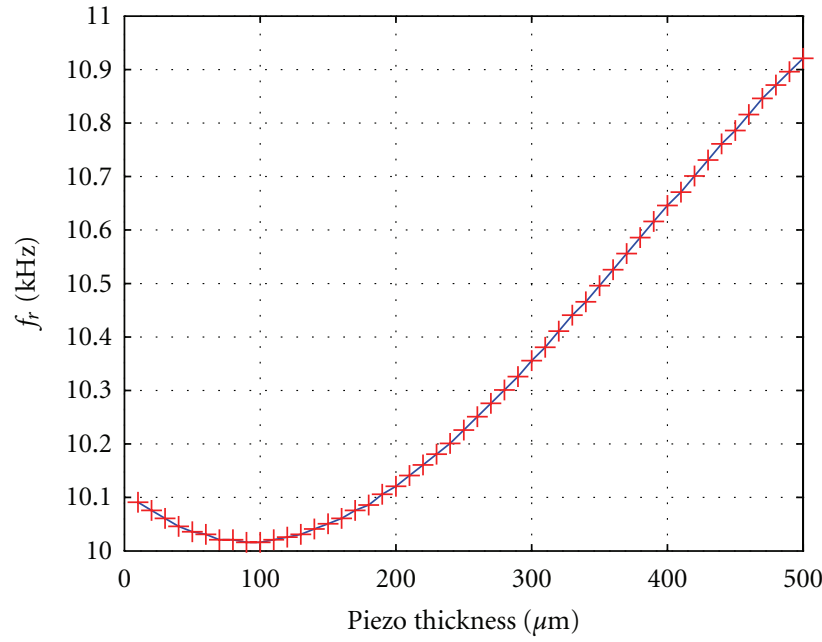

(b)

FIGURE 4: (a) First resonance frequency versus radius calculated for a piezoelectric layer thickness of 80 um. (b) First resonance frequency versus piezoelectric thickness calculated for a radius of $1 \mathrm{~cm}$.

be plotted for each radius or thickness, extending the data presented in Figure 3 to that shown in Figure 4, where radii ranged from 7.5 to $12.5 \mathrm{~mm}$ and thicknesses ranged from 10 to 500 micron.

Plotting the data of Figure 4(a) against inverse radius, a straight line is found (Figure 5), which means that the transducer first resonance frequency is inversely proportional to the radius, corroborating the findings of [22] which mean that these diaphragms would behave more as membranes
( $R^{-1}$ resonance frequency dependence) than as thin plates ( $R^{-2}$ resonance frequency dependence).

Three sample transducer frequency responses for radii of 8.3, 9.7, and $12.5 \mathrm{~mm}$ and piezoelectric thicknesses of 0.08 , 220, and 500 micron are shown in Figure 6.

Depending on the values of transducer radii or piezoelectric thicknesses, the resonance peaks in transducer FRF magnitude might increase more or less with respect to each other. To have a complete picture of how radii and thicknesses affect 
TABLE 2: Main parameters calculated after $s p$ and $a c$ analyses versus each of the independent variables: frequency, piezoelectric layer thickness, and transducer radius, keeping in each case two of them constant from values $f=10 \mathrm{kHz}, t_{\mathrm{PZE}}=80 \mathrm{um}$, and $R=1 \mathrm{~cm}$.

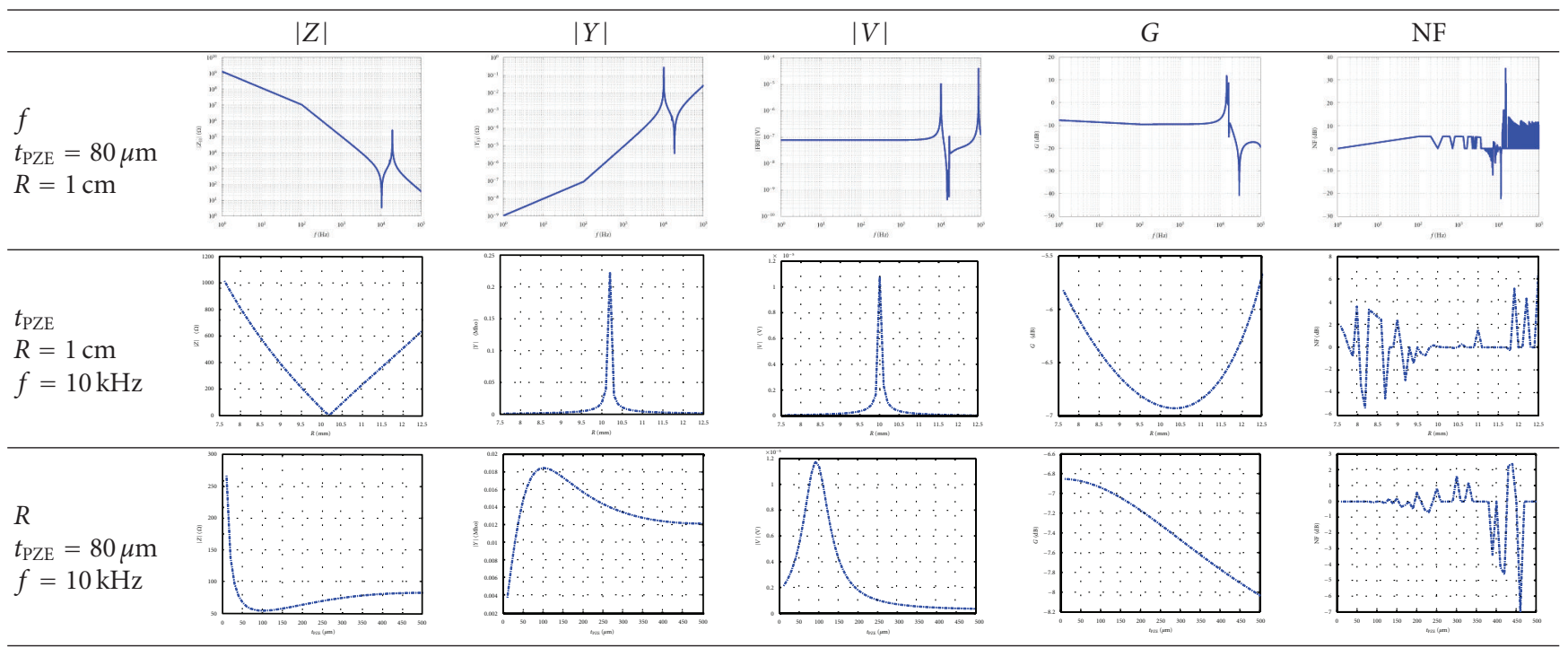

the impedance or transducer frequency response peaks, 3D plots are presented in Figures 7 and 8 at four different frequencies of 5, 10, 15, and $20 \mathrm{kHz}$.

Noise figure and transducer gain are characterized in a similar manner: in the case of noise figure (NF), for a fixed radius of $1 \mathrm{~cm}$, the values of NF versus frequency were obtained from the Cadence design environment for each piezoelectric layer thickness and are shown as a 3D plot in Figure 9. In the case of transducer gain, its calculated values from the Cadence design environment are similarly shown against frequency for a fixed radius of $1 \mathrm{~cm}$ and for each piezoelectric thickness (Figure 10).

\section{Discussion}

The results presented characterise the transducer design space and yield information and details on impedance, resonance frequency, noise figure, and transducer gain as a function of frequency, piezoelectric layer thickness, and transducer radius.

The main variables considered in this study induce to treat the problem as a 4-variable problem: frequency $(f)$, piezoelectric layer thickness $\left(t_{\mathrm{PZE}}\right)$, radius $(R)$, and one other variable for the $z$-axis which can be either frequency response $(V)$, impedance $(Z)$, admittance $(Y)$, noise figure (NF), or transducer gain $(G)$.

Therefore, 3D plots can be made of any of the $z$-axis variables with respect to two other independent variables after fixing the remaining one. For example, a 3D plot can be obtained of $|Z|$ versus piezoelectric thickness and transducer radius at any fixed frequency. Similarly, if transducer radius was fixed, the plot of $|Z|$ versus piezoelectric thickness and frequency could be produced. Alternatively, two variables might be fixed and the remaining two can be plotted against each other, which yield a plot matrix. Table 2 shows an

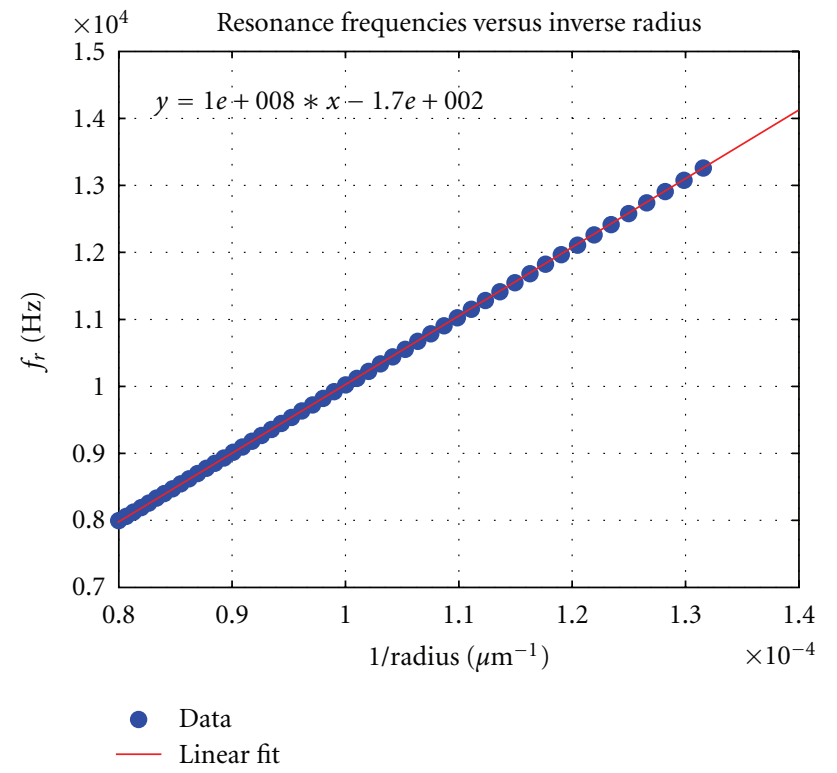

FIGURE 5: Diaphragm first resonance frequency versus inverse radius.

example of a matrix plot obtained for fixed values of $f=$ $10 \mathrm{kHz}, R=1 \mathrm{~cm}$, and $t_{\mathrm{PZE}}=80 \mu \mathrm{m}$.

This approach introduces a complete way of characterizing the piezoelectric transducer in terms of impedance, frequency response, admittance, transducer gain, and noise figure. Any two independent variable values might be set and the relation with the two others might be obtained numerically. After the design space is characterised the next logical step would be to introduce a multiobjective optimization of the transducer design variables. This approach will hopefully help improve the process steps involved in micromachining and manufacturing this kind of piezoelectric micro speakers. 


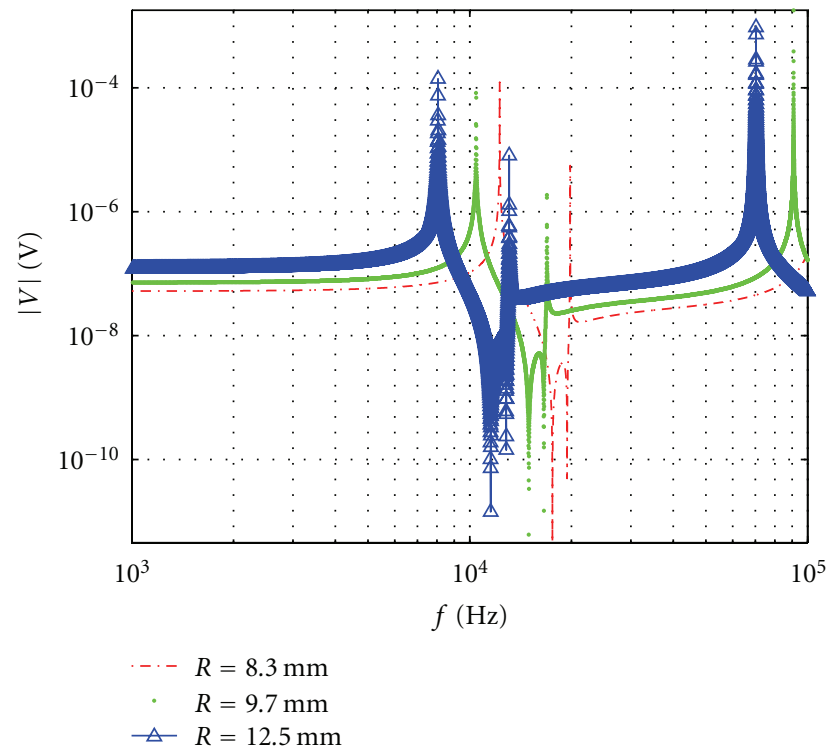

(a)

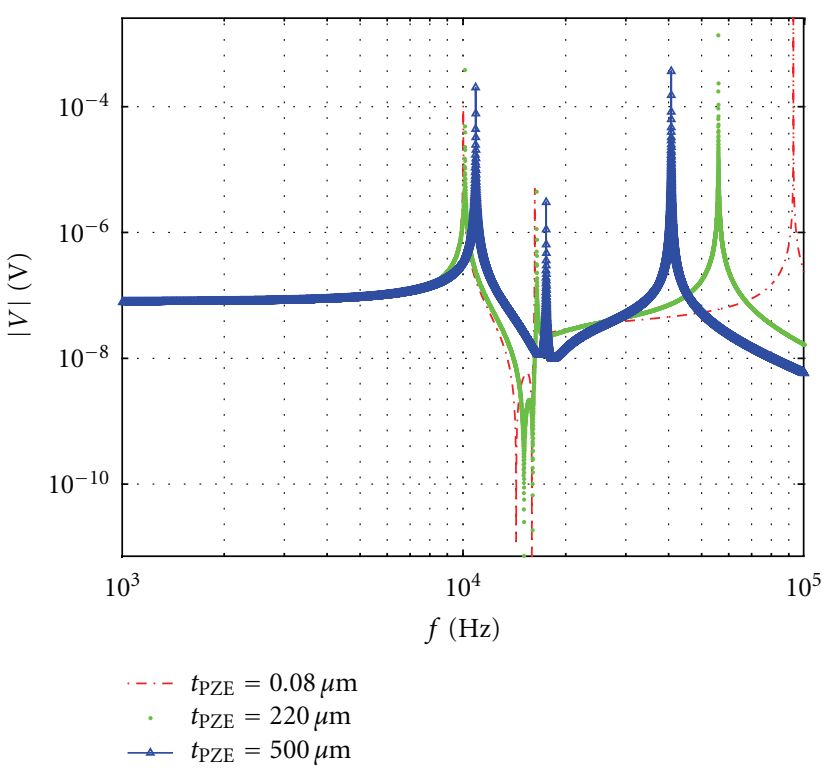

(b)

Figure 6: (a) Transducer electromechanical frequency response magnitudes for different radii. (b) Transducer electromechanical frequency response magnitudes for different piezoelectric layer thicknesses.

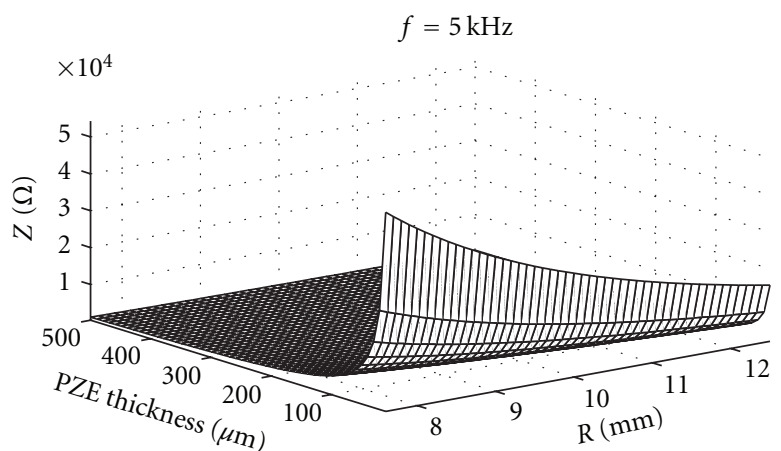

(a)

$$
f=15 \mathrm{kHz}
$$

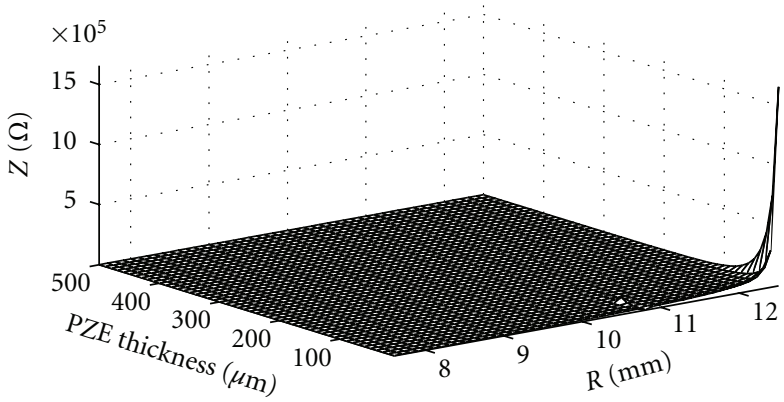

(c)

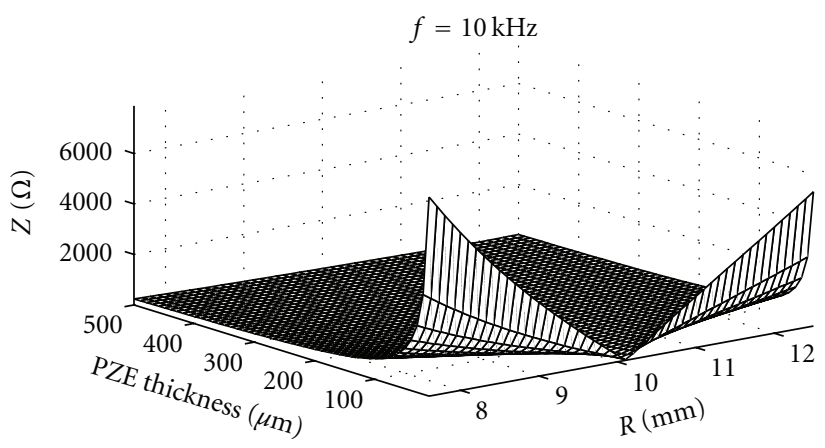

(b)

$$
f=20 \mathrm{kHz}
$$

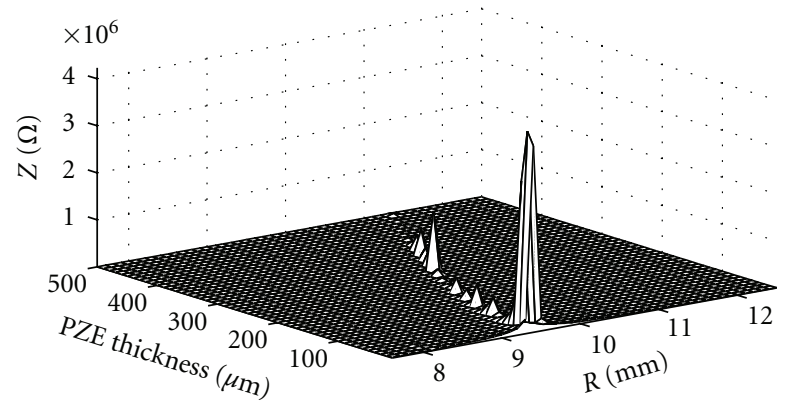

(d)

Figure 7: Peaks of impedance versus radius and piezoelectric layer thickness at four fixed frequencies. 


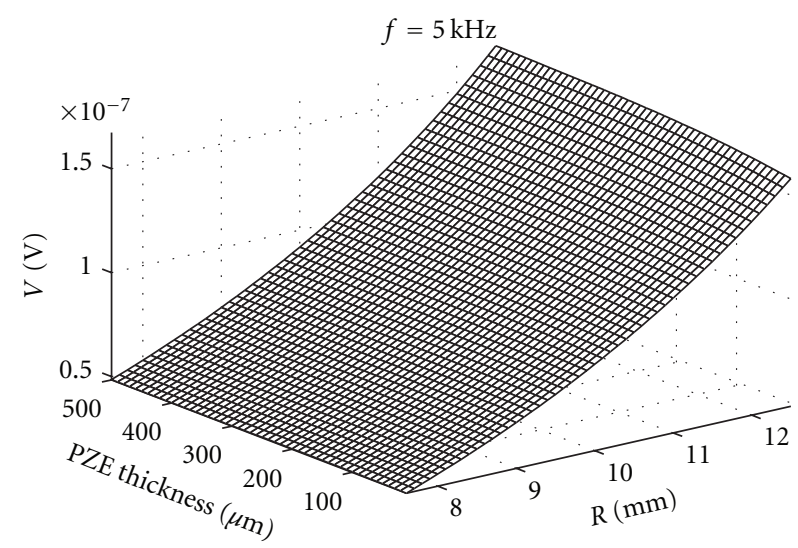

(a)

$$
f=15 \mathrm{kHz}
$$

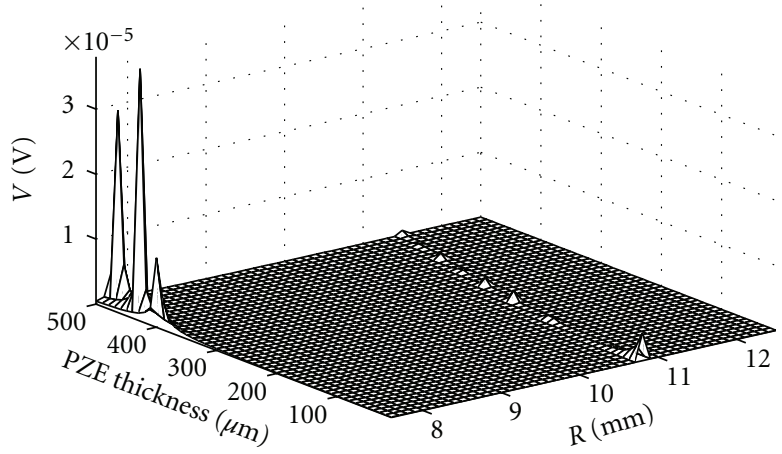

(c)

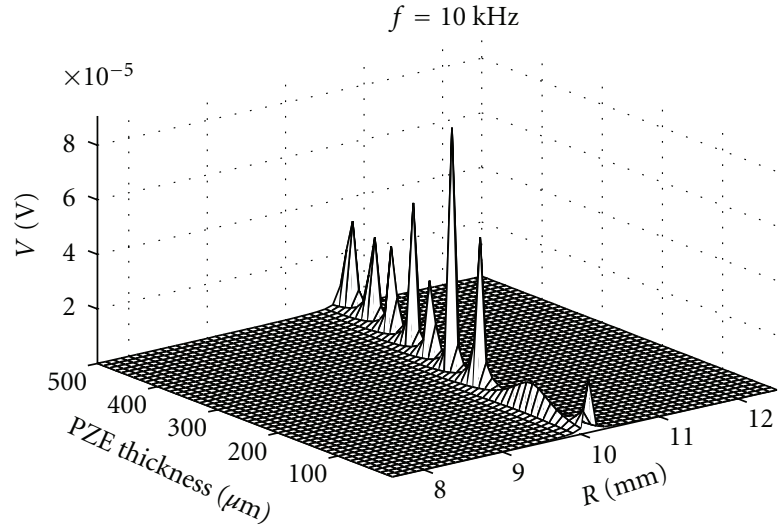

(b)

$$
f=20 \mathrm{kHz}
$$

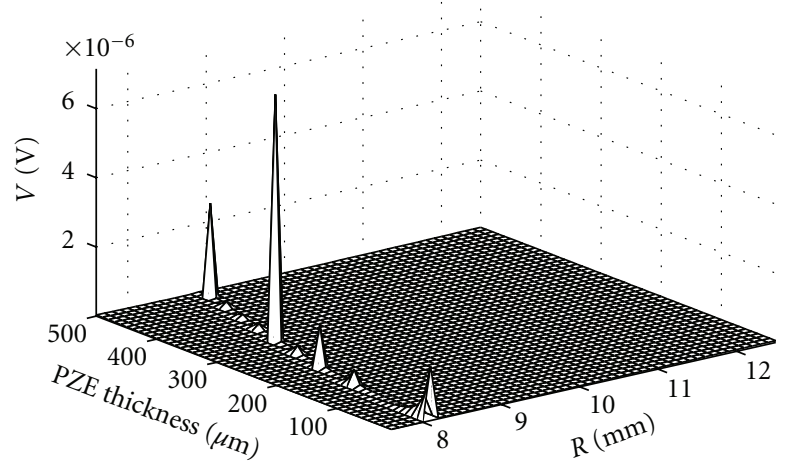

(d)

FIGURE 8: First resonance in FRF magnitude versus radius and piezoelectric layer thickness at four fixed frequencies.

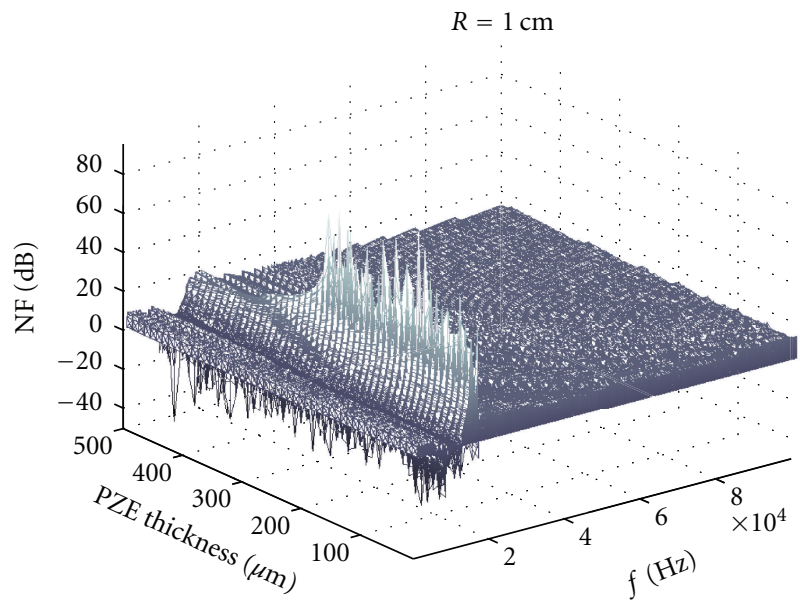

FIGURE 9: Noise figure versus frequency and piezoelectric thickness at fixed radius $=1 \mathrm{~cm}$.

\section{Conclusion}

The design and analysis of a piezoelectric micro speaker based on pMUT operation modelling and characterization is presented. The analysis is based on s-parameter analysis

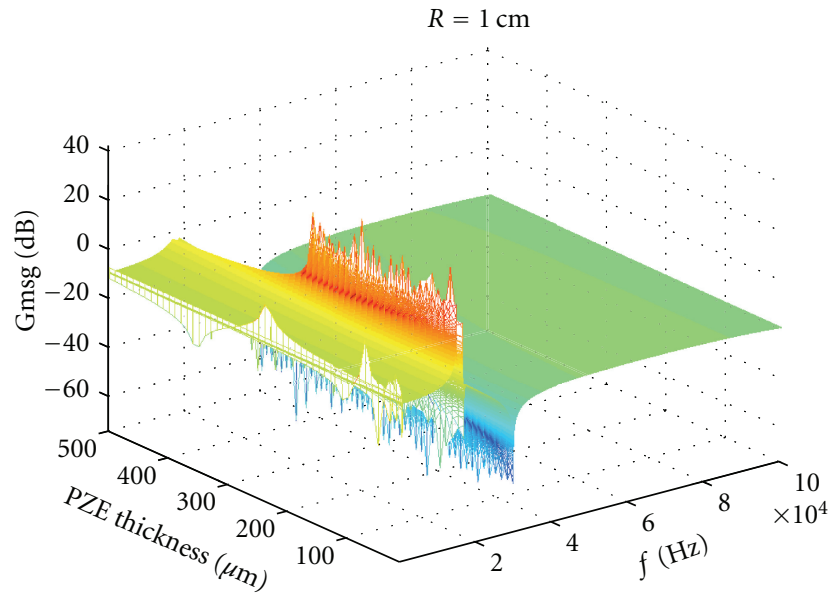

FIGURE 10: Maximum stable power gain versus frequency and piezoelectric thickness at fixed radius $=1 \mathrm{~cm}$.

for a two-port network, after which values for impedance, admittance, frequency response, noise figure, and transducer gain have been presented. Relations for transducer radius, piezoelectric thickness, and operating frequency were obtained which yield diaphragm displacement and therefore 
sound pressure level and noise figure values. Results represent useful tradeoffs for piezoelectric micro speaker design.

\section{Acknowledgments}

This work has been partially supported by the ESA project COSMIC VISION and by the Spanish Department of Science and Technology Project TEC2008-04920. J. Mendoza-López is grateful to partial funding by the postdoctoral program JAE-DOC granted by the Spanish National Research Council (Consejo Superior de Investigaciones Científicas, CSIC).

\section{References}

[1] C.-M. Lee and S.-M. Hwang, "Optimization of SPL and THD performance of microspeakers considering coupling effects," IEEE Transactions on Magnetics, vol. 47, no. 5, pp. 934-937, 2011.

[2] C. M. Lee, J. H. Kwon, K. S. Kim, J. H. Park, and S. M. Hwang, "Design and analysis of microspeakers to improve sound characteristics in a low frequency range," IEEE Transactions on Magnetics, vol. 46, no. 6, Article ID 5467616, pp. 2048-2051, 2010.

[3] J. H. Kwon, S. M. Hwang, and K. S. Kim, "Development of slim rectangular microspeaker used for minimultimedia phones," IEEE Transactions on Magnetics, vol. 43, no. 6, pp. 2704-2706, 2007.

[4] S. M. Hwang, H. J. Lee, K. S. Hong, B. S. Kang, and G. Y. Hwang, "New development of combined permanent-magnet type microspeakers used for cellular phones," IEEE Transactions on Magnetics, vol. 41, no. 5, pp. 2000-2003, 2005.

[5] M. R. Bai, C. Y. Liu, and R. L. Chen, "Optimization of microspeaker diaphragm pattern using combined finite elementlumped parameter models," IEEE Transactions on Magnetics, vol. 44, no. 8, Article ID 4618654, pp. 2049-2057, 2008.

[6] W. Klippel, "Nonlinear large-signal behavior of electrodynamic loudspeakers at low frequencies," Journal of the Audio Engineering Society, vol. 40, pp. 483-496, 1992.

[7] W. Klippel, "Dynamic measurement and interpretation of the nonlinear parameters of electrodynamic loudspeakers," Journal of the Audio Engineering Society, vol. 38, no. 12, pp. 944955, 1990.

[8] S. Yi, M. Yoon, and S. Ur, "Piezoelectric microspeakers with high compressive $\mathrm{ZnO}$ film and floating electrode," Journal of Electroceramics, vol. 23, no. 2-4, pp. 295-300, 2009.

[9] B. Belgacem, F. Calame, and P. Muralt, "Piezoelectric micromachined ultrasonic transducers based on PZT films," in Proceedings of the 15th IEEE International Symposium on Applications of Ferroelectrics (ISAF'06), August 2006.

[10] S. C. Ko, Y. C. Kim, S. S. Lee, S. H. Choi, and S. R. Kim, "Micromachined piezoelectric membrane acoustic device," Sensors and Actuators A, vol. 103, no. 1-2, pp. 130-134, 2003.

[11] D. H. Pearce, A. Hooley, and T. W. Button, "On piezoelectric super-helix actuators," Sensors and Actuators A, vol. 100, no. 2-3, pp. 281-286, 2002.

[12] K. A. Seffen, "Analysis of smart linear piezoelectric transducers," Smart Structures and Materials, vol. 3985, pp. 660-671, 2000.

[13] T. Pedersen, T. Zawada, K. Hansen, R. Lou-Moeller, and E. Thomsen, "Fabrication of high-frequency pMUT arrays on silicon substrates," IEEE Transactions on Ultrasonics, Ferroelectrics, and Frequency Control, vol. 57, no. 6, Article ID 5480189, pp. 1470-1477, 2010.
[14] J. L. Vernet, W. Steichen, R. Lardat, O. Garcia, and J. F. Gelly, "PMUTS design optimization for medical probes applications," in Proceedings of the IEEE Ultrasonics Symposium, pp. 899-902, October 2001.

[15] D. J. Morris, R. F. Need, M. J. Anderson, and D. F. Bahr, "Enhanced actuation and acoustic transduction by pressurization of micromachined piezoelectric diaphragms," Sensors and Actuators A, vol. 161, no. 1-2, pp. 164-172, 2010.

[16] R. A. Dorey, F. Dauchy, D. Wang, and R. Berriet, "Fabrication and characterization of annular thickness mode piezoelectric micro ultrasonic transducers," IEEE Transactions on Ultrasonics, Ferroelectrics, and Frequency Control, vol. 54, no. 12, Article ID 4430024, pp. 2462-2467, 2007.

[17] C. Wang, Z. Wang, T. L. Ren et al., "A micromachined piezoelectric ultrasonic transducer operating in d 33 mode using square interdigital electrodes," IEEE Sensors Journal, vol. 7, no. 7, pp. 967-977, 2007.

[18] J. Liu, C. Oakley, and R. Shandas, "Capacitive micromachined ultrasonic transducers using commercial multi-user MUMPs process: capability and limitations," Ultrasonics, vol. 49, no. 8, pp. 765-773, 2009.

[19] C. B. Doody, X. Cheng, C. A. Rich, D. F. Lemmerhirt, and R. D. White, "Modeling and characterization of CMOS-fabricated capacitive micromachined ultrasound transducers," Journal of Microelectromechanical Systems, vol. 20, pp. 104-118, 2010.

[20] IEEE Standard on Piezoelectricity ANSI/IEEE Std 176-1987, IEEE Ultrasonics, Ferro-electrics, and Frequency Control Society, 1987.

[21] S. A. N. Prasad, Q. Gallas, S. Horowitz et al., "Analytical electroacoustic model of a piezoelectric composite circular plate," AIAA Journal, vol. 44, no. 10, pp. 2311-2318, 2006.

[22] E. Hong, S. Trolier-McKinstry, R. Smith, S. V. Krishnaswamy, and C. B. Freidhoff, "Vibration of micromachined circular piezoelectric diaphragms," IEEE Transactions on Ultrasonics, Ferroelectrics, and Frequency Control, vol. 53, no. 4, pp. 697705, 2006.

[23] G. Anderson, "On the determination of finite integral transforms for forced vibrations of circular plates," Journal of Sound and Vibration, vol. 9, no. 1, pp. 126-144, 1969.

[24] S. Kopuz, Y. S. Ünlüsoy, and M. Çalişkan, "Integrated FEM/ BEM approach to the dynamic and acoustic analysis of plate structures," Engineering Analysis with Boundary Elements, vol. 17, no. 4, pp. 269-277, 1996.

[25] J. W. S. Rayleigh and R. B. Lindsay, The Theory of Sound, MacMillan, 1894.

[26] P. M. Morse and K. U. Ingard, Theoretical Acoustics, McGrawHill, 1968. 

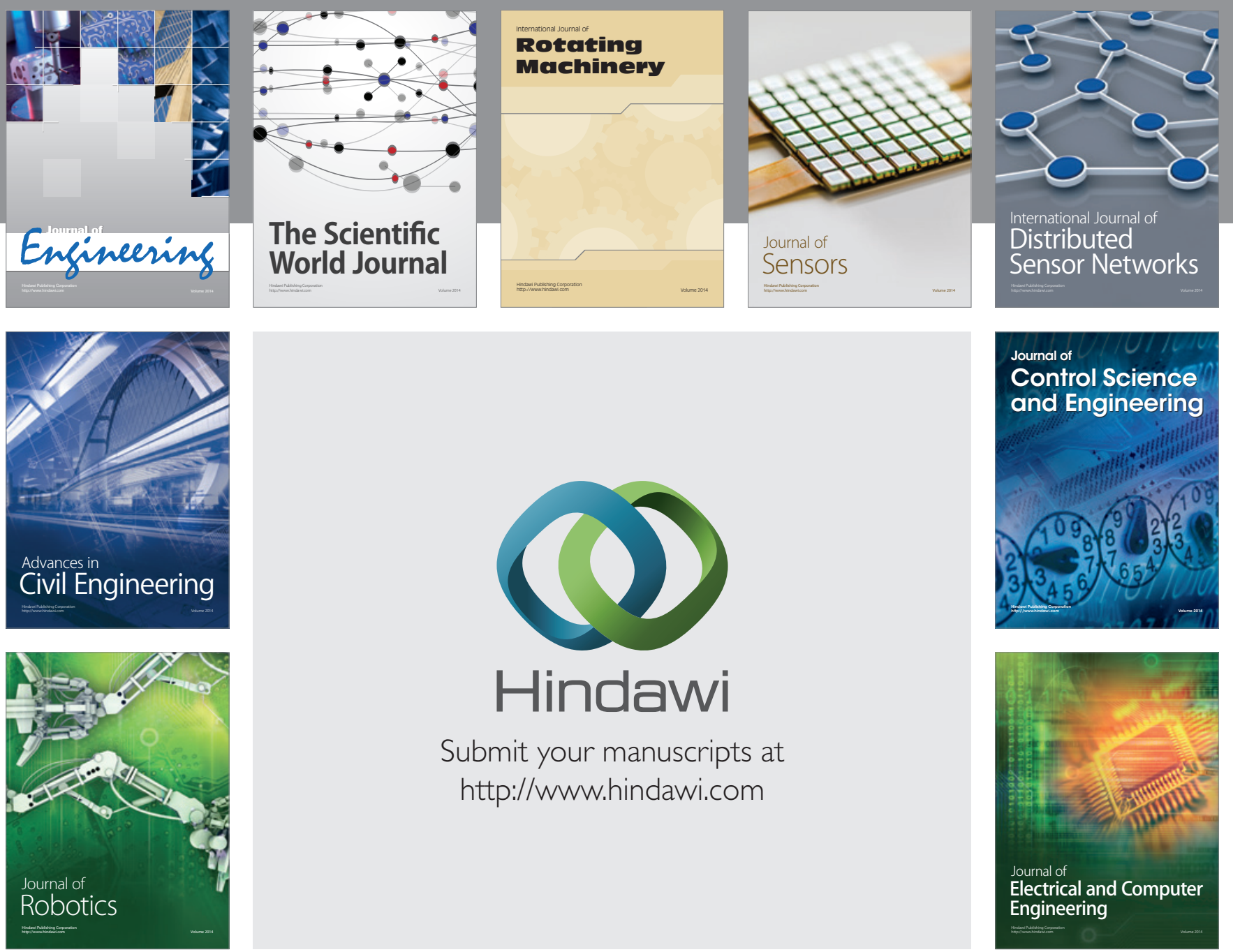

Submit your manuscripts at

http://www.hindawi.com
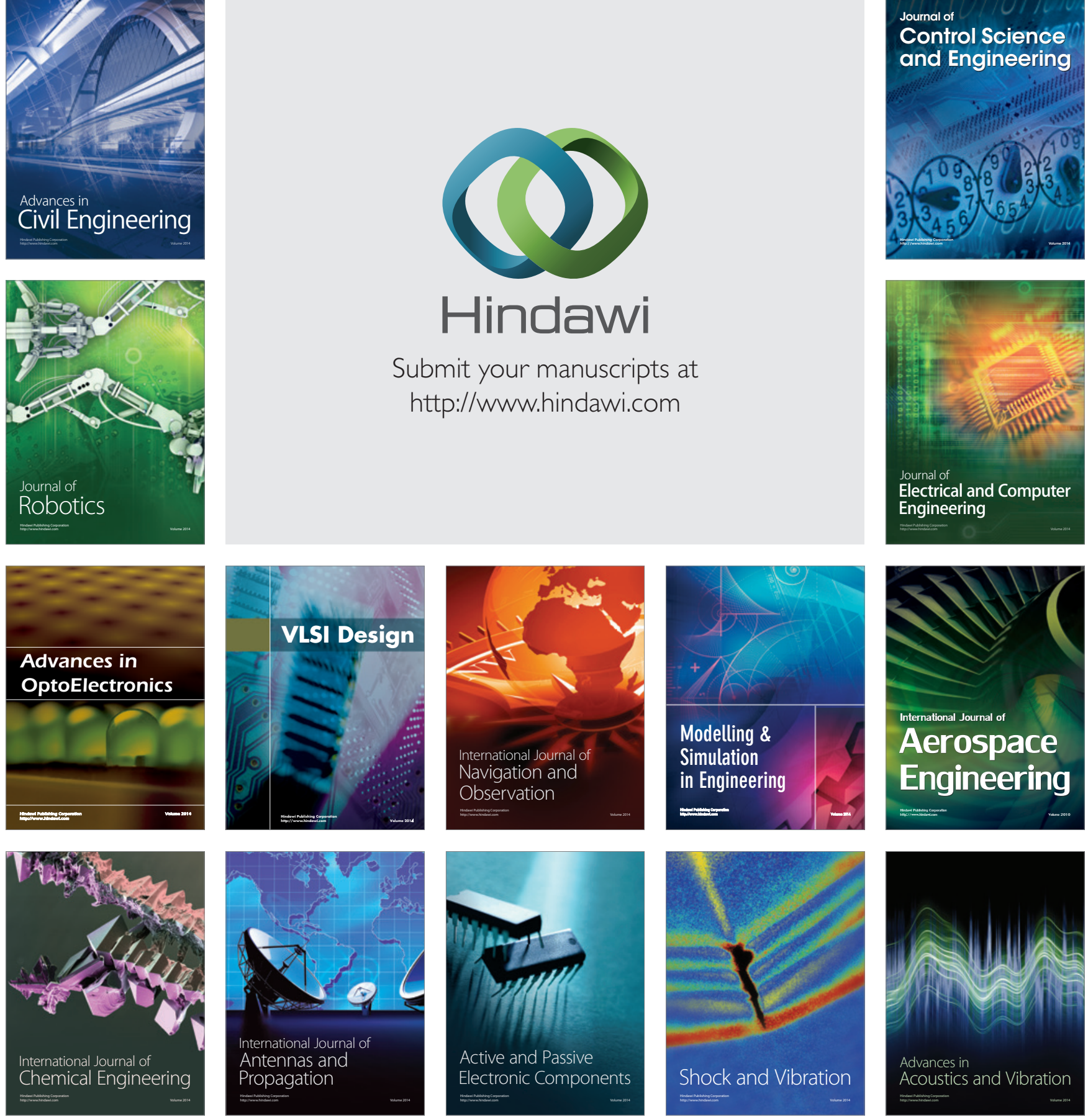